\title{
Nanocomposite hydrogels: an emerging biomimetic platform for myocardial therapy and tissue engineering
}

\author{
"The demand for new class of clinical therapies for cardiovascular \\ diseases is rapidly rising and these encouraging results offer a \\ promising future."
}

\begin{abstract}
Keywords: cardiovascular therapy • hydrogel $\bullet$ nanohybrids $\bullet$ regenerative medicine - tissue engineering
\end{abstract}

\section{Repairing damaged heart muscles: the challenges}

Recent years have seen a significant rise in patient survival rate after myocardial infarction, commonly known as heart attack. This is mainly due to improvement in hospital instrument facilities, clinical management system, surgical tools and technologies. However, many of these patients soon develop heart failure, with $50 \%$ mortality rate within 5 years of heart attack [1]. As the regeneration power of the heart muscle is very limited, negative remodeling and nonfunctional scar tissue deposition leads to gradual terminal failure of the heart. Current treatments include heart transplantation and ventricular assist devices, which are both highly invasive and risky surgical procedures with inconsistent results. Stem cell transplantation has recently shown some potential that can improve heart function through several mechanisms such as cardiac differentiation, neoangiogenesis and paracrine effects [2]. Despite promising results, stem cell therapy still remains limited because of economic, timing and logistic issues, particularly when isolating cells from elderly patients under clinical settings. Reduced cell survival and retention at the transplant site further limits their therapeutic potential [3]. Thus, we see an urgent need for new strategies to attenuate the progression of the disease postmyocardial infarction. The following sections highlight the emerging roles of biocompatible nanomaterials and hydrogels and provide insights for further development in cardiac therapy and tissue engineering applications.

\section{Nanomaterials \& injectable} hydrogels: how they can help repair damaged cardiac tissues

Although still in its early stage of development, the field of nanomedicine for cardiovascular therapy has evolved rapidly over the years with new discoveries in nanomaterials science [4]. In particular, local intramyocardial delivery of therapeutic nanoparticles, including lipopolymers and viral nanocarriers, has shown impressive in vivo results to provide early cardioprotection and enhance neovascularization of injured tissue [5-8]. However, retention of the implanted nanotherapeutics at the target site for an optimal duration is critical for the success of the therapy. And this is particularly challenging when delivering therapeutics to cardiac environment where the wall of the heart is constantly beating driving substantial amount of cargo to other nonessential regions.

Injectable hydrogel that helps retain the nanotherapeutics at the target site may offer a viable solution to this problem. Compared to other procedures, hydrogel with compatible rheological properties aids in reducing the damage to the surrounding tissues along with the added capability to preserve

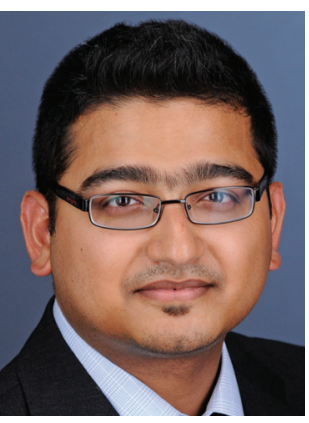

Arghya Paul Department of Chemical \& Petroleum Engineering, Bioengineering Graduate Program, School of Engineering, University of Kansas, 4165C Learned Hall 1530 W 15th St, Lawrence, KS 66045, USA arghyapaul@ku.edu 
the encapsulated drug at injected site. In general, therapeutic approaches of injectable hydrogels mainly include epicardial and endocardial injections. Main benefits of such delivery methods is that they allow direct access to the damaged tissue site, accurate placement and retention of the therapeutics compared with other methods. Injectable extracellular matrix (ECM) hydrogel from Ventrix Inc. (CA, USA), which is now going for clinical trial for myocardial therapy, is one such example [9]. Importantly, the liquid hydrogel precursor can be delivered in a single bolus or in multiple injection forms, where the later can subsequently get transitioned in situ into hydrogel state by shift in salt concentration, $\mathrm{pH}$, temperature, or enzyme concentration. In case of bio-originated materials, such as decellularized ECM matrix, the hydrogel can additionally provide structural support and biochemical cues to the injured site which may induce endogenous stem cell homing for natural healing.

"...the cardiac and stem cell originated in vitro cardiac tissue prototypes have demonstrated that they can help better understand the cell-drug interactions, drug resistance and cytotoxicity, pharmacological profiling and instigate personalized medicine practice."

A smart selection of such drug carrying nanoparticles and injectable hydrogel can complement each other's strengths and help develop the next generation therapeutics with multifunctional effects. Recently, it has been reported that DNA carrying nanomaterials in combination with naturally derived methacrylated gelatin (GelMA) hydrogel, can efficiently deliver DNA to the infarcted site when administered intramyocardially [10]. We also noticed that $V E G F$ gene-activated nano graphene oxide (GO), suspended in injectable GelMA, can effectively deliver $V E G F$ genes to the myocardial tissue in peri-infarct region which eventually leads to neovascularization, attenuation of cardiac dysfunction and scar formation. In fact GelMA alone, as a major ECM derivative, exhibit potential to induce functional benefits to the tissues because of its inherent biological cues. miRNA analysis of the tissues at injected site further confirmed the biocompatibility of the developed nanocomposite GelMA/GO hydrogel. Other studies have shown beneficial effects of injectable shear thinning hydrogels to deliver therapeutics to target site by its shear-induced flow properties commonly induced by syringes and catheters [11,12]. The shear-thinning materials recover back into hydrogel state immediately after delivery and retain the encapsulated therapeutics at the target site. Purcell et al., in an interesting study, demonstrated that an on- demand matrix metalloproteinase (MMP) inhibition is possible using such injectable bioresponsive hydrogel which releases recombinant tissue inhibitors of MMPs only when MMP activity is increased postmyocardial infarction [13]. In recent years, several other bioresponsive materials for cardiac applications have been developed which, in combination with nanovectors, can deliver oxygen-releasing agents, small molecules, drugs and growth factors at injured site [14,15]. The technology can be further improved using nanoparticles which can bind to specific target sites. For this the nanoparticles must be surface functionalized with high density ligand molecules which can identify a substantially overexpressed molecular target at the injured tissue site. It is also important to mention here that these nanohybrids can be surgically implanted at any site in patients with minimal prior knowledge of the geometry of the injured site.

\section{Engineering miniaturized cardiac tissues:} role of biomimetic nanocomposite hydrogels The last few years have seen significant thrust in designing in vitro miniaturized healthy and diseased cardiac tissue models also known as 'heart on-a-chip' [16,17]. To achieve this goal tissue engineering is now entering a new era where it merges the insights of microscale technologies with stem cell biology, in particular induced pluripotent stem cells. So far the cardiac and stem cell originated in vitro cardiac tissue prototypes have demonstrated that they can help better understand the cell-drug interactions, drug resistance and cytotoxicity, pharmacological profiling and instigate personalized medicine practice. Moreover, such bioengineered cardiac constructs can also be used as cardiac patches for functional tissue replacement therapy. However, one of the main challenges to develop such a clinically relevant tissue engineered cardiac structure is replicating the unique morphological, physiological and functional properties of the native myocardium.

In a classical approach, cardiac cells are combined with hydrogels, and cultivated in perfusable bioreactors which provide mechanical and electrical stimulation. Yet, poor conductive nature of commonly used biomaterials result in cardiac constructs with suboptimal performance. Incorporation of electroconductive nanomaterials such as carbon nanotubes (CNTs) and GO nanosheets can potentially solve this problem. CNT-reinforced hybrid GelMA hydrogel has been shown to improve mechanical and electrical properties of hydrogel [18]. The resulting nanocomposite hydrogel significantly improved cardiomyocyte alignment, maturation and electrophysiological functions. Developing such biomimetic nanocomposite hydrogels can facilitate proper electroconductive network and estab- 
lish spontaneous beating behavior within the cardiac cells. Toward the same goal Dvir et al. demonstrated that the addition of gold nanowires and nanoparticles to biomaterial-based hydrogels can significantly enhance electrical communication between neighboring cardiac cells in a 3D microenvironment $[19,20]$. As a result, the adjacent cardiac cells exhibited a synchronous response to electrical pacing and generated proper excitation-contraction coupling with each beat. Thus it can be stated that the ability to fabricate functional cardiac tissues depends on engineering hydrogels which closely represents cardiac cell niche and biomimetic microarchitechture.

\section{Future perspective}

It can be envisaged from the recent studies articulated in this editorial that nanohybrid hydrogels can be highly useful for cardiac tissue engineering and therapeutics. Although new nanocomposite biomaterials are consistently being developed, we need to prioritize these materials as per biomedical applications. Studies with generally-recognized-as-safe materials and hydrogels will have the greatest chances for clinical translation. However, several challenges need to overcome before considering them fit for clinical realization. This includes inventing methods to vascularize the engineered cardiac tissues, optimization

\section{References}

Papers of special note have been highlighted as:

- of interest; $\bullet$ of considerable interest

1 Go AS, Mozaffarian D, Roger VL et al. Heart disease and stroke statistics - 2013 update: a report from the American Heart Association. Circulation 127(1), e6-e245 (2013).

2 Chong JJ, Yang X, Don CW et al. Human embryonic-stemcell-derived cardiomyocytes regenerate non-human primate hearts. Nature 510 (7504), 273-277 (2014).

3 Paul A, Ge Y, Prakash S, Shum-Tim D. Microencapsulated stem cells for tissue repairing: implications in cell-based myocardial therapy. Regen. Med. 4(5), 733-745 (2009).

4 Donaldson K, Duffin R, Langrish JP et al. Nanoparticles and the cardiovascular system: a critical review. Nanomedicine 8(3), 403-423 (2013).

5 Chang MY, Yang YJ, Chang CH et al. Functionalized nanoparticles provide early cardioprotection after acute myocardial infarction. J. Control. Release 170(2), 287-294 (2013).

6 Zsebo K, Yaroshinsky A, Rudy JJ et al. Long-term effects of AAV1/SERCA2a gene transfer in patients with severe heart failure: analysis of recurrent cardiovascular events and mortality. Circ. Res. 114(1), 101-108 (2014).

7 Paul A, Hasan A, Rodes L, Sangaralingam M, Prakash S. Bioengineered baculoviruses as new class of therapeutics using micro and nanotechnologies: principles, prospects and challenges. Adv. Drug Deliv. Rev. 71, 115-130 (2014). of microfabrication technique to consistently develop identical cardiac constructs for high-throughput screening and drug discovery applications. Other concerns related to application of nanocomposite injectable hydrogel include long-term biosafety of the implants, easy integrateability of hydrogel to host tissue and knowledge about fate of implanted nanomaterials postimplantation. While these are few of the many parameters that need to be investigated in designing nanocomposite hydrogels for specific cardiac applications, clearly many unique opportunities and challenges await equally for the clinicians and academic research communities. The demand for new class of clinical therapies for cardiovascular diseases is rapidly rising and these encouraging results offer a promising future.

\section{Financial \& competing interests disclosure}

The author would like to acknowledge the Institutional Development Award (IDeA) from the National Institute of General Medical Sciences, NIH (P20 GM103638). The author has no other relevant affiliations or financial involvement with any organization or entity with a financial interest in or financial conflict with the subject matter or materials discussed in the manuscript apart from those disclosed.

No writing assistance was utilized in the production of this manuscript.

8 Harel-Adar T, Ben Mordechai T, Amsalem Y, Feinberg MS, Leor J, Cohen S. Modulation of cardiac macrophages by phosphatidylserine-presenting liposomes improves infarct repair. Proc. Natl Acad. Sci. USA 108(5), 1827-1832 (2011).

9 Seif-Naraghi SB, Singelyn JM, Salvatore MA et al. Safety and efficacy of an injectable extracellular matrix hydrogel for treating myocardial infarction. Sci. Transl. Med. 5(173), 173ra125 (2013).

- Paper reports a new injectable extracellular matrix hydrogel that repairs damaged tissue after heart attack in the porcine model.

10 Paul A, Hasan A, Kindi HA et al. Injectable graphene oxide/hydrogel-based angiogenic gene delivery system for vasculogenesis and cardiac repair. ACS Nano 8(8), 8050-8062 (2014).

-. Reports a new class of injectable graphene oxide-based nanocomposite hydrogel which can be successfully used for in vivo local delivery of therapeutics to myocardially infarcted rat heart.

11 Gaharwar AK, Avery RK, Assmann A et al. Shear-thinning nanocomposite hydrogels for the treatment of hemorrhage. ACS Nano 8(10), 9833-9842 (2014).

12 Wong Po Foo CT, Lee JS, Mulyasasmita W, Parisi-Amon A, Heilshorn SC. Two-component protein-engineered physical hydrogels for cell encapsulation. Proc. Natl Acad. Sci. USA 106(52), 22067-22072 (2009). 
13 Purcell BP, Lobb D, Charati MB et al. Injectable and bioresponsive hydrogels for on-demand matrix metalloproteinase inhibition. Nat. Mater. 13(6), 653-661 (2014).

14 Li Z, Guo X, Guan J. An oxygen release system to augment cardiac progenitor cell survival and differentiation under hypoxic condition. Biomaterials 33(25), 5914-5923 (2012).

15 Oh KS, Song JY, Yoon SJ, Park Y, Kim D, Yuk SH. Temperature-induced gel formation of core/shell nanoparticles for the regeneration of ischemic heart. J. Control. Release 146(2), 207-211 (2010).

16 Bhatia SN, Ingber DE. Microfluidic organs-on-chips. Nat. Biotechnol. 32(8), 760-772 (2014).

17 Agarwal A, Goss JA, Cho A, Mccain ML, Parker KK. Microfluidic heart on a chip for higher throughput pharmacological studies. Lab Chip 13(18), 3599-3608 (2013).
- Represents the first high-throughput 'heart on a chip' microfluidic in vitro system which can alleviate the translational and commercial barriers of current systems by improving the scalability and reproducibility.

18 Shin SR, Jung SM, Zalabany M et al. Carbon-nanotubeembedded hydrogel sheets for engineering cardiac constructs and bioactuators. ACS Nano 7(3), 2369-2380 (2013)

19 Shevach M, Fleischer S, Shapira A, Dvir T. Gold nanoparticle-decellularized matrix hybrids for cardiac tissue engineering. Nano Lett. 14(10), 5792-5796 (2014).

20 Dvir T, Timko BP, Brigham MD et al. Nanowired threedimensional cardiac patches. Nat. Nanotechnol. 6(11), 720-725 (2011).

-. First study to demonstrate that electroconductive nanocomposite hydrogels can be used to enhance the structure, phenotype and function of engineered cardiac tissue. 